\title{
Selection and validation of miRNAs as normalizers for profiling expression of microRNAs isolated from thyroid fine needle aspiration smears
}

\author{
SERGEI E. TITOV ${ }^{1,2}$, PAVEL S. DEMENKOV ${ }^{3,4}$, MIKHAIL K. IVANOV ${ }^{1,2}$, EKATERINA S. MALAKHINA ${ }^{5}$, \\ TATIANA L. POLOZ ${ }^{6}$, ELENA V. TSIVLIKOVA ${ }^{2}$, MARIA S. GANZHA ${ }^{2}$, SERGEI P. SHEVCHENKO ${ }^{7}$, \\ LYUDMILA F. GULYAEVA ${ }^{8}$ and NIKOLAY N. KOLESNIKOV ${ }^{1}$
}

\begin{abstract}
${ }^{1}$ Institute of Molecular and Cellular Biology, Siberian Branch of the Russian Academy of Sciences, Novosibirsk 630090; ${ }^{2}$ AO Vector-Best, Novosibirsk 630117; ${ }^{3}$ The Federal Research Center Institute of Cytology and Genetics, Siberian Branch of the Russian Academy of Sciences, Novosibirsk 630090; ${ }^{4}$ Novosibirsk State University, Novosibirsk 630090; ${ }^{5}$ Institute of Chemical Biology and Fundamental Medicine, Siberian Branch of the Russian Academy of Sciences, Novosibirsk 630090; ${ }^{6}$ Non-governmental Healthcare Institution 'Railroad Clinical Hospital on the Station Novosibirsk-Glavny', Joint Stock Company 'Russian Railways', Novosibirsk 630003; ${ }^{7}$ Novosibirsk Municipal Budgetary Healthcare Institution 'Municipal Clinical Hospital \#1', Novosibirsk 630047; ${ }^{8}$ Institute of Molecular Biology and Biophysics, Siberian Branch of the Russian Academy of Medical Sciences, Novosibirsk 630117, Russia
\end{abstract}

Received April 29, 2016; Accepted September 1, 2016

DOI: $10.3892 /$ or.2016.5113

\begin{abstract}
Fine needle aspiration cytology (FNAC) is currently the method of choice for malignancy prediction in thyroid nodules. Nevertheless, in some cases the interpretation of FNAC results may be problematic due to limitations of the method. The expression level of some microRNAs changes with the development of thyroid tumors, and its quantitation can be used to refine the FNAC results. For this quantitation to be reliable, the obtained data must be adequately normalized. Currently, no reference genes are universally recognized for quantitative assessments of microRNAs in thyroid nodules. The aim of the present study was the selection and validation of such reference genes. Expression of 800 microRNAs in 5 paired samples of thyroid surgical material corresponding to different histotypes of tumors was analyzed using NanoString technology and four of these (hsa-miR-151a-3p, -197-3p, $-99 a-5 p$ and $-214-3 p$ ) with the relatively low variation coef-
\end{abstract}

Correspondence to: Dr Sergei E. Titov, Institute of Molecular and Cellular Biology, Siberian Branch of the Russian Academy of Sciences, Acad. Lavrentiev Avenue 8/2, Novosibirsk 630090, Russia E-mail: titovse78@gmail.com

Abbreviations: FNAC, fine-needle aspiration cytology; Cq, quantification cycle; RT-qPCR, quantitative reverse-transcription real-time PCR; FA, follicular adenoma; PTC, papillary thyroid carcinoma; MTC, medullary thyroid carcinoma; ND, non-diagnostic material; NF, normalization factor

Key words: microRNA profiling, thyroid cancer, fine needle aspiration cytology, normalization, reference genes ficient were selected. The possibility of use of the selected microRNAs and their combination as references was estimated by RT-qPCR on a sampling of cytological smears: benign $(n=226)$, atypia of undetermined significance $(n=9)$, suspicious for follicular neoplasm $(n=61)$, suspicious for malignancy $(n=19)$, medullary thyroid carcinoma (MTC) $(n=32)$, papillary thyroid carcinoma (PTC) $(n=54)$ and non-diagnostic material (ND) $(n=34)$. In order to assess the expression stability of the references, geNorm algorithm was used. The maximum stability was observed for the normalization factor obtained by the combination of all 4 microRNAs. Further validation of the complex normalizer and individual selected microRNAs was performed using 5 different classification methods on 3 groups of FNAC smears from the analyzed batch: benign neoplasms, MTC and PTC. In all cases, the use of the complex classifier resulted in the reduced number of errors. On using the complex microRNA normalizer, the decision-tree method C4.5 makes it possible to distinguish between malignant and benign thyroid neoplasms in cytological smears with high overall accuracy (>91\%).

\section{Introduction}

Thyroid lesions are the commonest endocrine pathologies in the human population. Palpation of thyroid gland enables the detection of nodules in 5-7\% of the examined patients, ultrasound screening in $20-50 \%$ (1). Of those, only approximately $5 \%$ are malignant and require surgical intervention. Benign nodules do not become cancerous (2), and it is, therefore, important to establish the nodule type at an early stage in order to select the appropriate treatment. The basic method to discriminate malignant from benign nodules is fine needle aspiration cytology (FNAC). This method requires highly 
experienced and skilled staff, which makes it prone to errors due to the human factor (3). The greatest difficulty is associated with follicular thyroid cancer (FTC) that cannot cytologically be differentiated from follicular adenoma (FA). Therefore, a serious need exists to enhance the accuracy of preoperative thyroid lesion typing. For this purpose, molecular markers (oncogenic somatic mutations, the level of oncogene expression and the methylation status of regulatory regions of certain genes) may be used (4). Recent studies have shown that some microRNAs (miRNAs) can serve as such markers, because their levels are significantly changed in a wide variety of tumors, including thyroid tumors (5-8). miRNAs are more stable than mRNAs and are characterized by better stability in routine procedures for FNAC preparations and storage of samples. Therefore, the miRNA content can be measured directly in material from dried FNAC smears, thus, avoiding additional manipulations and supplementing routine cytology study with the results of miRNA expression analysis.

Currently, quantitative reverse-transcription real-time PCR (RT-qPCR) is one of the most frequently used methods for the quantitative assessment of the level of gene expression, because of its sensitivity and relatively low cost of reagents and instrumentation as compared to alternative approaches (RNA-Seq, Microarrays and NanoString). The reliable registration of the changes in miRNA levels by means of RT-qPCR requires correct normalization of the 'raw' data. The purpose of normalization is to minimize the variation in the results due to technical reasons. The main sources of the variation are the amount, degradation rate and the purity of the RNA preparation. There are different approaches to addressing the problem of normalization, but none of those is conventional for miRNA quantitation (9). The common element of all employed normalization strategies is to use the reference genes, i.e. genes whose expression does not differ or minimally varies in the compared types of tissues or cells (10). The simplest approach to estimate the expression stability of reference genes is seeking the minimum variability in the quantification cycles of PCR (Cq) (11) between the samples. Such an approach, however, is only effective when concentration of cDNA derived from different samples is equal or at least close. Equalization of cDNA concentration in the sample by adding the equal starting amounts of total RNA does not solve this problem. Total RNA is mostly represented by ribosomal RNA, which ratio to mRNA and especially to miRNA can vary significantly in different samples $(10,12)$. In 2002 Vandesompele et al (13) described the algorithm (called geNorm) for finding stably expressed reference genes. This method makes it possible to rank candidate reference genes by their expression stability, based on the calculation of an average pairwise variation between all studied genes and to determine the optimum set of reference genes required for normalization.

One of the primary tasks of any investigation involving gene expression is the selection of the appropriate reference gene(s). Currently in most cases there are no universally recognized reference genes or their combinations for miRNA expression analysis in the specific tissue type. For miRNA expression profiling it is desirable that reference genes also coded miRNAs because in technical terms (extraction efficiency, RT-PCR and storage stability) they are similar to
miRNAs of interest and all experimental procedures affect them in a similar way. A single gene may be used for normalization (14), but it has been demonstrated that such strategy may lead to serious errors while the use of multiple reference genes significantly enhances the measurement accuracy $(9,13)$. Vandesompele et al (13) proposes to use at least three reference genes for proper normalization. In some cases, small nuclear (RNU6, RNU44 and RNU48) or nucleolar (snoRNA202 and snoRNA234) RNAs are used as a universal normalizer instead of miRNAs. This approach may bring about systemic errors, for example, there is recent evidence suggesting that the levels of expression of small nuclear RNAs may also change significantly during carcinogenesis $(15,16)$.

Since to the best of our knowledge, there are still no universally accepted reference miRNAs, which could be used as normalizers for miRNA quantification in FNAC preparations, the present study was aimed at the selection and validation of such normalizers.

\section{Materials and methods}

All biological material was obtained in compliance with the legislation of the Russian Federation, and written informed consent was provided by all the patients, all the data was depersonalized. This study was approved by the ethics committee of the Institute of Molecular Biology and Biophysics, Siberian Branch of the Russian Academy of Medical Sciences. We used 435 cytological specimens obtained by a standard fine-needle aspiration biopsy in the Center of New Medical Technologies (Novosibirsk). The aspirated samples were smeared on a glass slide and processed in a standard way, i.e., the smear was airdried, the material was pre-fixed by $95 \%$ ethanol for $10 \mathrm{~min}$ and stained by hematoxylin and eosin. The smears were classified according to Bethesda system (17): non-diagnostic (ND) $(n=34)$, benign $(n=226)$, atypia of undetermined significance $(n=9)$, suspicious for follicular neoplasm $(n=61)$, suspicious for malignancy $(n=19)$, medullary thyroid carcinoma (MTC) $(n=32)$, papillary thyroid carcinoma (PTC) $(n=54)$. We also used samples of thyroid tumor tissue, surgically removed in 152 patients and representing different histotypes of neoplasms: 32 benign neoplasms, 33 FAs and 87 PTCs. Sample collection and histology analysis were controlled by a qualified oncologist (Novosibirsk Municipal Clinical Hospital \#1, Oncology Department VI).

Isolation of the total nucleic acids and detection of miRNAs and RNU6 by RT-qPCR was performed as previously described (18).

Evaluation of human DNA content was performed by qPCR as previously described (18), using oligos for the conserved region of chromosome 15 . The sequences of all new oligos missing in our previous study (18) are listed in Table I.

The expression of 800 miRNAs was evaluated using the nCounter Human v2 miRNA Expression Assay kit (NanoString Technologies, Seattle, WA, USA) in accordance with the producer's instructions. For the analysis, $100 \mathrm{ng}$ of total RNA, isolated from surgical material and measured by Qubit (Invitrogen, Waltham, MA, USA), was hybridized for $20 \mathrm{~h}$ at $65^{\circ} \mathrm{C}$. After hybridization, the samples were placed in an automatic nCounter Prep Station for purification and binding of reporter probes. Then every sample was scanned in 550 fields 
Table I. Sequences of oligonucleotides used in the study.

\begin{tabular}{|c|c|c|}
\hline Target & Type & Sequence $\left(5^{\prime}-3^{\prime}\right)$ \\
\hline miR-183 & $\begin{array}{l}\text { RT primer } \\
\text { R PCR primer } \\
\text { PCR probe } \\
\text { F PCR primer }\end{array}$ & $\begin{array}{l}\text { CCTGAGTCTGAGGCTCACTGAGACCTTTCGCACCCTCGACTCAGGCAGTGAATTC } \\
\text { CTGAGGCTCACTGAGACCT } \\
\text { (R6G)-TTCGCACCC(T-BHQ1)CGACTCAGGCAGTGAATTC-p } \\
\text { CGGACTATGGCACTGGTA }\end{array}$ \\
\hline $\operatorname{miR}-551 b$ & $\begin{array}{l}\text { RT primer } \\
\text { R PCR primer } \\
\text { PCR probe } \\
\text { F PCR primer }\end{array}$ & $\begin{array}{l}\text { GAGGAGAGGCCTTGTAGCACGACCTTATCCTCACCTCCTCTCCTCCTGAAACC } \\
\text { GCCTTGTAGCACGACCTTA } \\
\text { (R6G)-TC(C-LNA)TCACC(T-BHQ1)CCTCTCCTCCTGAAACC-p } \\
\text { CACACTCAGCGACCCATACTT }\end{array}$ \\
\hline miR-361 & $\begin{array}{l}\text { RT primer } \\
\text { R PCR primer } \\
\text { PCR probe } \\
\text { F PCR primer }\end{array}$ & $\begin{array}{l}\text { GTCGTGTCTGAGGCTCACTGAGGACTTCGCAGCGCTGACACGACGTACCCCT } \\
\text { CTGAGGCTCACTGAGACCT } \\
\text { (R6G)-TTCGCAGCGC(T-BHQ1)GACACGACGTACCC-p } \\
\text { CAGCCGTTATCAGAATC(T-LNA)CC }\end{array}$ \\
\hline $\operatorname{miR}-151 \mathrm{a}$ & $\begin{array}{l}\text { RT primer } \\
\text { R PCR primer } \\
\text { PCR probe } \\
\text { F PCR primer }\end{array}$ & $\begin{array}{l}\text { CGTGATGCTGAGGCTCACTGAGACCTTTCGCACCCTCGCATCACGC(C-LNA)T(C-LNA)AAGG } \\
\text { CTGAGGCTCACTGAGACCT } \\
\text { (R6G)-TTCGCACCC(T-BHQ1)CGCATCACGCCTCAAGG-p } \\
\text { ACAGGACCTAGACTGAAGCT }\end{array}$ \\
\hline miR-197 & $\begin{array}{l}\text { RT primer } \\
\text { R PCR primer } \\
\text { PCR probe } \\
\text { F PCR primer }\end{array}$ & $\begin{array}{l}\text { GTCGTGGGTGAAGCAGACAGACACAATTACGCACCTGCCACGACGCTGGGTG } \\
\text { GTGAAGCAGACAGACACAA } \\
\text { (R6G)-TTACGCACC(T-BHQ1)GCCACGACGCTGGGTG-p } \\
\text { CCACGTTCACCACCTTCTC }\end{array}$ \\
\hline miR-99a & $\begin{array}{l}\text { RT primer } \\
\text { R PCR primer } \\
\text { PCR probe } \\
\text { F PCR primer }\end{array}$ & $\begin{array}{l}\text { CGTGATGCTGAGGCTCACTGAGACCTTTCGCACCCTCGCATCACGCACAAGATC } \\
\text { CTGAGGCTCACTGAGACCT } \\
\text { (R6G)-TTCGCACCC(T-BHQ1)CGCATCACGCACAAGATC-p } \\
\text { TAGGACACCCGTAGATCCG }\end{array}$ \\
\hline $\operatorname{miR}-214$ & $\begin{array}{l}\text { RT primer } \\
\text { R PCR primer } \\
\text { PCR probe } \\
\text { F PCR primer }\end{array}$ & $\begin{array}{l}\text { GTACGTGCGCTCTGCTGACACCACTCTATCCTACCCTCGCACGTACACTGCCT } \\
\text { GCTCTGCTGACACCACTCTA } \\
\text { (R6G)-TCCTACCC(T-BHQ1)CGCACGTACACTGCCTG-p } \\
\text { ATACACAGCAGGCACAGA }\end{array}$ \\
\hline Hs-Ch15 & $\begin{array}{l}\text { F PCR primer } \\
\text { R PCR primer } \\
\text { PCR probe }\end{array}$ & $\begin{array}{l}\text { GGAGAGCCTAGGAGAATGTAT } \\
\text { AATCACTCTTCTGGAGGCA } \\
\text { (R6G)-TGGTTTCAGA(T-BHQ1)CTTCATGTTGGGTCTCCACGT-p }\end{array}$ \\
\hline
\end{tabular}

R6G, rhodamine 6G (rhodamine 590); BHQ1, black hole ouencher-1; LNA, locked nucleic acid. R, reverse; F, forward.

of view in nCounter Digital Analyzer. The data were analyzed using nSolver v2 software (NanoString Technologies). The results were normalized to 100 most abundant miRNAs.

The geometric mean of the quantification cycles of four miRNAs (NF4) was calculated by the formula (1):

$$
C q(N F 4)=\sqrt[4]{C q_{1} * C q_{2} * C q_{3} * C q_{4}}
$$

The most stable reference genes were selected using geNorm algorithm: let there be data on the expression $n$ of various miRNAs in $m$ samples, for each pair of miRNAs the vector $A_{j k}$ was calculated, the components of which are

$$
A_{j k}=\left\{\log _{2}\left(\frac{a_{1 j}}{a_{1 k}}\right), \log _{2}\left(\frac{a_{2 j}}{a_{2 k}}\right), \ldots, \log _{2}\left(\frac{a_{m j}}{a_{m k}}\right)\right\}, \forall j, k \in[1, n], k \neq j
$$

computed as logarithm to the base two of the ratio between the level of miRNA expression in a single sample (formula 2):

$$
V_{j k}=\sum_{k=1}^{n} \operatorname{st} . \operatorname{dev}\left(A_{j k}\right), k \neq j
$$

The pairwise variation $V_{j k}$ of miRNA defined as standard derivation of $A_{j k}$ elements (formula 3):

$$
M_{j}=\frac{\sum_{k=1}^{n} V_{j k}}{n-1}, k \neq j
$$

The expression stability of miRNA $\mathrm{j}\left(M_{j}\right)$ is the arithmetic mean of all pairwise variations $V_{j k}$ (formula 4):

Classification of thyroid cytological preparations by the levels of miRNA expression was performed using the program TANAGRA (19). The following five methods were selected: i) Linear discriminant analysis (20); ii) Naive Bayes 
Table II. P-values for pairwise compared subgroups of samples selected for the validation of the normalization factor (PTC, MTC and benign) and non-diagnostic smears.

\begin{tabular}{|c|c|c|c|c|c|}
\hline & \multicolumn{5}{|c|}{ P-values (Mann-Whitney U test) } \\
\hline & RNU6 & $\operatorname{miR}-197$ & miR-99a & $\operatorname{miR}-151 \mathrm{a}$ & $\operatorname{miR}-214$ \\
\hline $\mathrm{ND} /$ Benign & 0.850806 & 0.344236 & 0.110171 & 0.394331 & 0.911211 \\
\hline Benign/MTC & $0.000000^{\mathrm{c}}$ & 0.513717 & $0.014811^{\mathrm{a}}$ & $0.048389^{\mathrm{a}}$ & 0.366020 \\
\hline Benign/PTC & $0.000000^{\mathrm{c}}$ & $0.003504^{\mathrm{b}}$ & $0.000000^{c}$ & 0.115550 & $0.000000^{c}$ \\
\hline \multirow[t]{3}{*}{ MTC/PTC } & 0.313498 & 0.162672 & 0.964270 & $0.047874^{\mathrm{a}}$ & $0.000311^{\mathrm{c}}$ \\
\hline & \multicolumn{5}{|c|}{ Median Cq (Interquartile range) } \\
\hline & RNU6 & miR-197 & miR-99a & miR-151a & $\operatorname{miR}-214$ \\
\hline ND & $27.8(2.7)$ & $31.2(1.4)$ & $30.2(3.2)$ & $28.8(1.7)$ & $35.9(1.9)$ \\
\hline Benign & $28.3(2.2)$ & $31.1(1.8)$ & $29.2(2.5)$ & $28.5(1.7)$ & $35.8(3.0)$ \\
\hline MTC & $24.6(3.8)$ & $31.1(5.2)$ & $26.1(6.1)$ & $29.9(6.4)$ & $36.4(4.1)$ \\
\hline PTC & $25.4(3.4)$ & $30.5(2.1)$ & $26.4(3.5)$ & $28.5(2.7)$ & $33.5(3.5)$ \\
\hline
\end{tabular}

ND, non-diagnostic; MTC, medullary thyroid carcinoma; PTC, papillary thyroid carcinoma. ${ }^{\mathrm{a}} \mathrm{P}<0.05$; ${ }^{\mathrm{P}}<0.01$; ${ }^{\mathrm{c}} \mathrm{P}<0.001$. Median $\mathrm{Cq}$ values are presented for comparison.

classifier (21); iii) Multilayer perceptron (Rumelhart multilayer perceptron) (22); iv) C-SVC, support vector machine from the library libSVM (23); and v) C4.5 decision tree algorithm (24).

In order to assess the quality of prediction for a group of samples based on different methods cross-validation with 5 partitions was used.

\section{Results}

Selection of reference miRNAs and evaluation of expression stability of all miRNAs and RNU6. To the best of our knowledge, no validated reference genes have been identified for miRNA expression analysis in thyroid tissues. U6 small RNA (RNU6) is frequently used for this purpose (14), however, it represents a different class of molecules, i.e., its length is different from that of mature miRNA, is transcribed by different RNA polymerase and circulates in complexes with different proteins. Thus, it appears more appropriate to use reference miRNAs. Therefore, our first task was to select several candidate reference miRNAs. For this purpose, we used NanoString technique (25) to simultaneously evaluate the expression of 800 miRNAs in 10 paired samples of thyroid surgical material (from the nodule and adjacent non-tumor tissue from the same patient): PTC-1 sample, follicular variant of PTC-1; FTC-1, FA-1, benign nodule -1.

For 249 miRNAs valuable expression (notably higher than in the negative controls) was shown in majority of the samples, with the range of measured concentrations of $3.8 \mathrm{lg}$. The contribution of various factors to the overall measurement error using NanoString decreased in the series: the heterogeneity of the tissue preparation; procedure for RNA isolation; analytical variation in the assay (data not shown). We selected 93 miRNAs whose expression did not vary significantly between different types of tumors and between the tumor/ adjacent non-tumor tissue and which have been identified in all samples in the amount of $\geq 50$ copies per reaction, since at a lower concentration the method variation was significantly higher (data not shown). From this list, five miRNAs with lowest coefficient of variation were selected: hsa-miR-361-5p, $-151 a-3 p,-197-3 p,-99 a-5 p$ and -214-3p (further they are designated as miR-151a, 197, -99a, -214 and -361). Such miRNAs we will refer to as 'reference miRNAs', those used for normalization (their levels are not expected to vary significantly between different lesion types).

To estimate the relative stability, the expression of 5 candidate reference miRNAs was measured by RT-qPCR in 435 FNAC samples. In parallel, the expression of 8 miRNAs selected as classifiers (as well as RNU6) was measured in the same samples by the same method. By 'classifying miRNAs' we mean those used for classification of FNAC samples. These miRNAs were selected based on literature data (26-29). The list of classifiers, further used for building classification algorithms, included hsa-miR-146b-5p, -183-5p, -187-3p, -199b-5p, $-205-5 p,-221-3 p,-375$ and $-551 b-3 p$; hereinafter referred to as miR-146b, -183, -187, -199b, -205, -221, -375 and -551b. RNU6 was selected as commonly used for normalization in miRNA expression studies. The content of miR-361 in our samples was low (characteristic Cq values of 36-38), which resulted in unacceptably high analytical variation of measurements (data not shown). Therefore, this miRNA was excluded from the further analysis. The median $\mathrm{Cq}$, reflecting the content of all other markers in the analyzed sample and the variation of their values is presented in Fig. 1. Table II shows median of raw $\mathrm{Cq}$ values and the results of the pairwise comparison for groups of samples with different cytological classification.

It is clear from Fig. 1 and Table II that the content of all analyzed RNA, including reference ones, significantly varies in FNACs. This variation only partially can be referred to the different total amount of RNA in samples and method errors; a significant contribution thereto is made by biological 


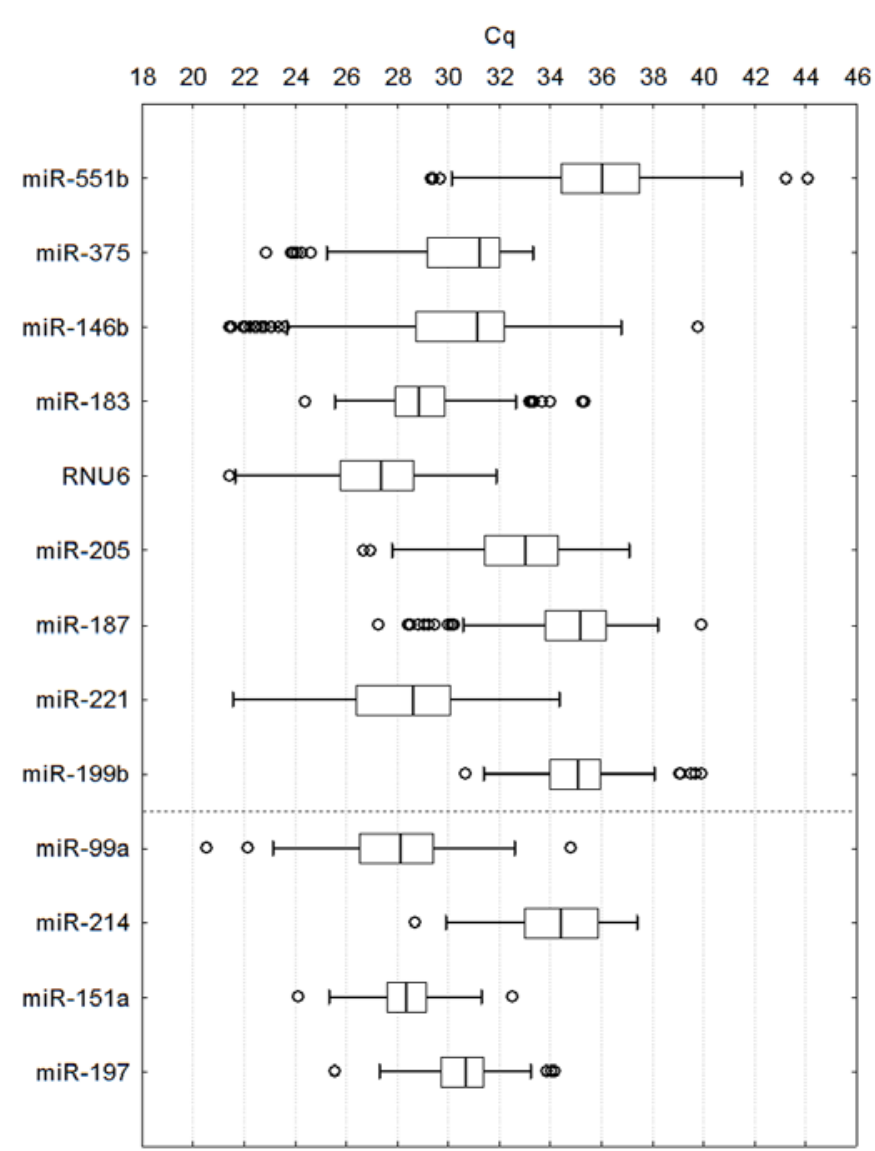

Figure 1. Box-whisker plot of $\mathrm{Cq}$ variation for reference and classifying short RNAs in the analyzed sample of 435 FNACs. For Cq values figure presents: inner line, the median value; box, upper and lower quartiles; whisker, nonoutlier range; outliers designated by circles. Horizontal dash line separates classifying (miR-551-199b) from reference (miR-99a-197) miRNAs.

differences between the samples, especially differences in the content of miRNAs between different subgroups.

For assessing the expression stability, we used the geNorm algorithm, which ranks the analyzed genes by the value of the relative expression stability denoted by M. Higher M-values are indicative of less stable expression because $M$ is an averaged pairwise variation value of a particular gene with all other genes (formula 4).

As expected, the expression stability of reference miRNAs appeared higher than of classifier miRNAs. At the same time, expression stability of RNU6 was found to be quite low; actually, it was higher for half of the miRNA classifiers (Fig. 2).

Selection of normalization factor. If the reference genes were considered separately, the lowest expression stability was observed for RNU6 and the highest - for miR-197; miR-99a, -151a and -214 had similar M-values. The optimal number of reference genes can be determined based on the V-value (pairwise variations $V_{n / n+1}$ between normalization factors) (formula 3): if the addition of extra reference genes into normalization factor (NF) leads to only a slight decrease in the $\mathrm{V}$-value, their use will not contribute significantly to reducing normalization errors (30).

The choice of the optimum number of reference miRNAs for the normalization is illustrated by the histogram showing

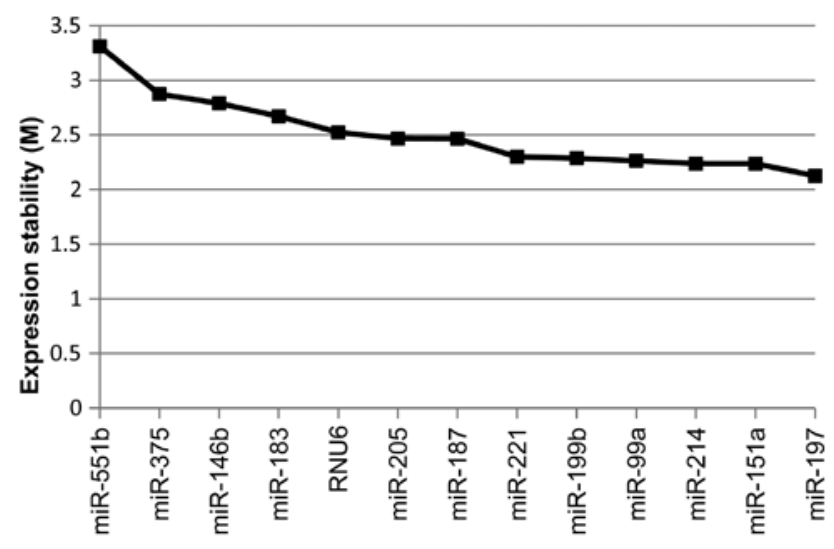

Figure 2. geNorm expression stability plot for analyzed miRNAs and RNU6.

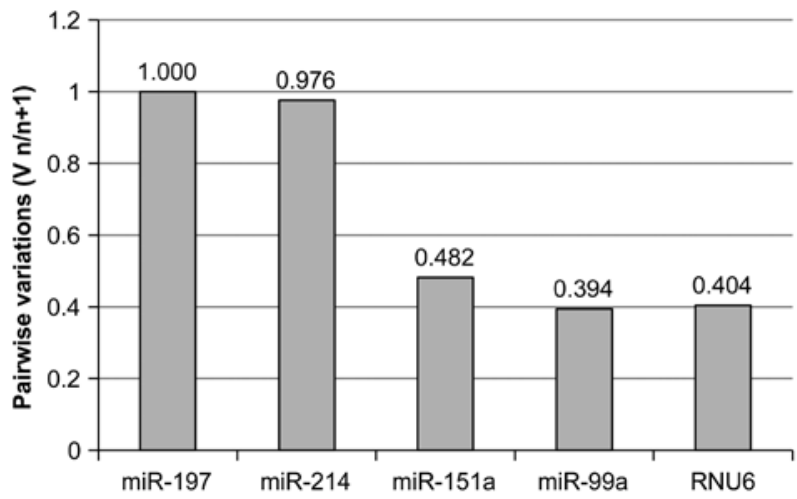

Figure 3. Determination of the optimal number of reference genes for normalization by geNorm analysis. Every bar represents the change in normalization accuracy when stepwise adding more reference genes according to the ranking in Fig. 2.

the changes in the variation when an additional miRNA is included into the NF (Fig. 3). That is, for instance, the column 'miR-214' on the histogram presents the ratio of the data variation under the normalization to miR-197 and to the geometric mean of miR-197+miR-214. It was proposed to use geometric rather than arithmetic mean by Vandesompele et al (13), because it is less affected by a wide scatter of values.

If the addition of a reference gene reduces the variation ratio in the normalization factor, it is reasonable to include this gene into NF. It is recommended that the number of genes in NF should be at least three (13). In our case, for normalization we selected miR-197, -151a, -214 and -99a. Addition of RNU6 to the NF no longer reduced data variation but, on the contrary, increased it (Fig. 3).

Therefore, the normalization factor (NF4) selected by us is a geometric mean of expression data of four miRNAs: miR-197, -214, -151a and -99a. Its expression stability was higher than that of every single reference miRNA (Fig. 4A).

One of the specific features of dried FNAC samples is high variability in quantity and degree of degradation of nucleic acids in the preparations. Thus, the Cq values for human DNA in our sampling could differ by 14 (which corresponds to the difference of $\sim 4$ orders of magnitude in the concentration) with interquartile range of one order of magnitude and the coefficient of variation $(\mathrm{Cv})$ of 6.7. We assessed the expression 
A

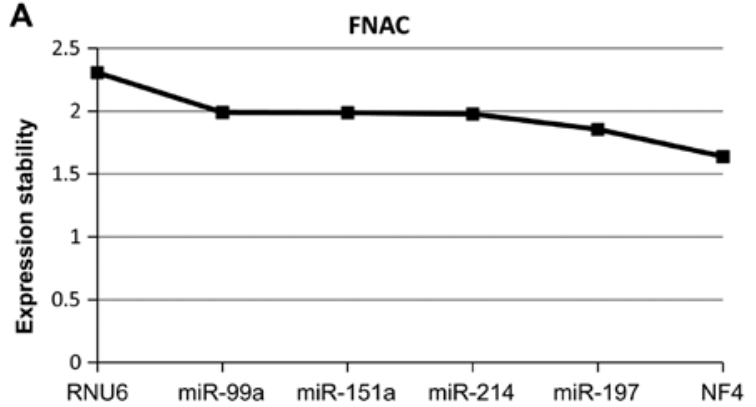

B

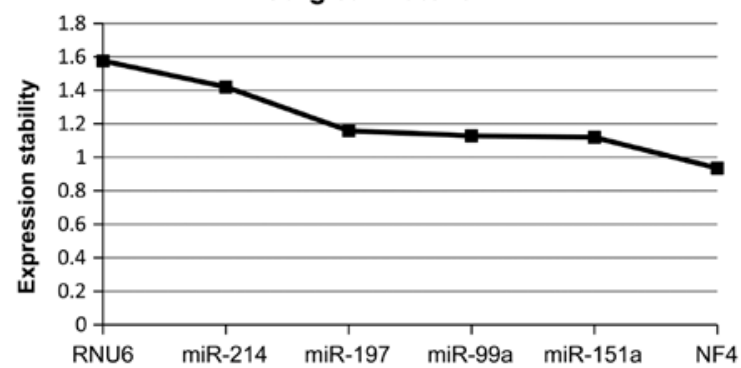

Figure 4. (A) Expression stability of reference miRNAs, RNU6, and NF4 (geometric mean of miR-197, -151a, -214 and -99a) obtained for FNAC smears. (B) The same for the surgical material.

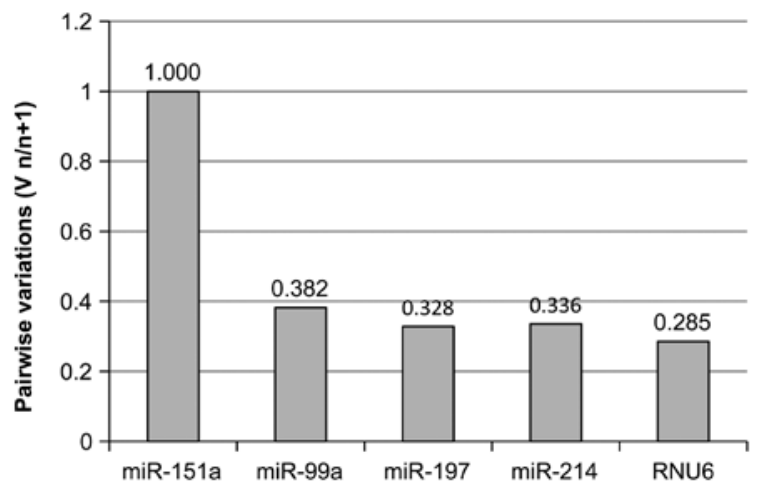

Figure 5. Pairwise variations between the normalization factors NFn and $\mathrm{NF}(\mathrm{n}+1)$ in the analysis of surgical material.

stability of reference miRNAs, RNU6 and NF4 for the surgical material obtained from patients with different types of thyroid neoplasms. For these samples, variation in the content of nucleic acids was significantly lower (the difference between the maximum and minimum concentrations of human DNA was 2 orders of magnitude, interquartile range was 4 times and $\mathrm{Cv}=4.8)$. As expected, the $\mathrm{M}$-values for reference miRNAs in the surgical material were lower than for FNACs (Fig. 4B), and, in contrast to those and with the exception of RNU6, met the criteria of stability, according to the recommendations of the developers of geNorm algorithm. It can be seen that both in FNACs and surgical material, the minimum stability is observed for RNU6 and the maximum stability is attained for $\mathrm{NF} 4$, however, the relative stability of these normalizers varies in samples corresponding to different material.

Histogram in the Fig. 5 illustrates the change in the variation observed when the same four miRNAs and RNU6 are
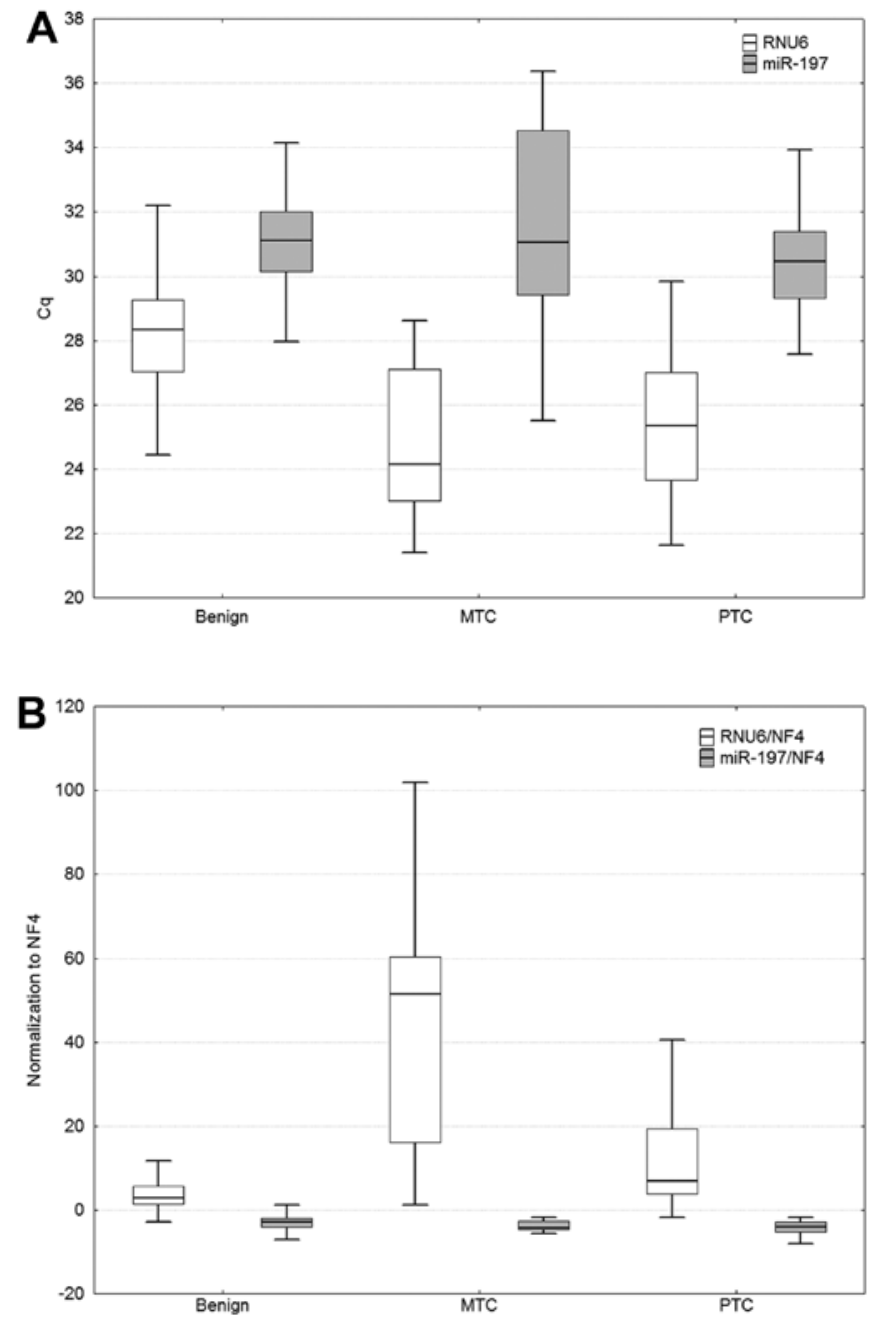

Figure 6. The content of RNU6 and miR-197 in FNACs, corresponding to different types of neoplasms. (A) Raw quantification cycles; (B) Cq values normalized to NF4.

used for normalization in the analysis of surgical material. Obviously, the variation is noticeably lower for this material as compared with FNACs; the relative contributions made to it by different normalizers vary, and the inclusion of RNU6 in the normalization somewhat enhances the stability, if only slightly. The differences between the two types of materials with regard to RNU6 can be attributed at least to two reasons. First, the relative stability of RNU6 and miRNAs in cytological smears could differ. Secondly, our sampling of FNACs, in contrast to the surgical material, was enriched by medullary cancers which are relatively rare. Fig. 6 presents the differences in the content of RNU6 in three groups of FNACs representing different neoplasm types. It can be seen that MTC samples differ significantly in the content of RNU6 $\left(\mathrm{P}=2 \times 10^{-11}\right)$.

Validation of the NF4. In order to validate the NF4, we selected 3 groups of FNACs, i.e., benign neoplasms, medullary thyroid carcinomas and papillary thyroid carcinomas. Such groups were selected because they include uniform data (benign or malignant tumors), other groups: atypia of undetermined significance, follicular neoplasm, suspicious for a follicular neoplasm and suspicious for malignancy, can include both benign and malignant tumors. We normalized data on the 
Table III. Comparison of error levels of different classification methods.

Papillary carcinoma/Medullary carcinoma/Benign

\begin{tabular}{|c|c|c|c|c|c|c|c|c|c|}
\hline & \multirow{2}{*}{\multicolumn{3}{|c|}{ Normalization to NF4 }} & \multirow{2}{*}{\multicolumn{3}{|c|}{ Normalization to miR-197 }} & \multirow{2}{*}{\multicolumn{3}{|c|}{ Normalization to miR-99a }} \\
\hline & & & & & & & & & \\
\hline & $\begin{array}{c}\text { Error } \\
(\%)\end{array}$ & $\begin{array}{c}\text { Cross- } \\
\text { validation } \\
\text { error }(\%)\end{array}$ & $\begin{array}{c}\text { Accuracy } \\
(\%)\end{array}$ & $\begin{array}{c}\text { Error } \\
(\%)\end{array}$ & $\begin{array}{c}\text { Cross- } \\
\text { validation } \\
\text { error }(\%)\end{array}$ & $\begin{array}{c}\text { Accuracy } \\
(\%)\end{array}$ & $\begin{array}{l}\text { Error } \\
(\%)\end{array}$ & $\begin{array}{c}\text { Cross- } \\
\text { validation } \\
\text { error }(\%)\end{array}$ & $\begin{array}{c}\text { Accuracy } \\
(\%)\end{array}$ \\
\hline $\mathrm{C} 4.5$ & 0.68 & 6.14 & 93.86 & 4.07 & 9.10 & 90.90 & 2.71 & 8.34 & 91.66 \\
\hline Support vector machine & 7.46 & 9.59 & 90.41 & 14.92 & 16.07 & 83.93 & 19.66 & 20.21 & 79.79 \\
\hline Multilayer perceptron & 3.05 & 4.28 & 95.72 & 3.73 & 8.90 & 91.10 & 6.78 & 11.24 & 88.76 \\
\hline Bayes classifier & 6.10 & 7.52 & 92.48 & 13.22 & 13.72 & 86.28 & 17.63 & 18.83 & 81.17 \\
\hline \multirow[t]{4}{*}{ Linear discriminant analysis } & 5.42 & 6.90 & 93.10 & 11.19 & 12.14 & 87.86 & 16.95 & 18.55 & 81.45 \\
\hline & \multicolumn{9}{|c|}{ Malignant neoplasms/Benign } \\
\hline & \multicolumn{3}{|c|}{ Normalization to NF4 } & \multicolumn{3}{|c|}{ Normalization to miR-197 } & \multicolumn{3}{|c|}{ Normalization to miR-99a } \\
\hline & $\begin{array}{c}\text { Error } \\
(\%)\end{array}$ & $\begin{array}{c}\text { Cross- } \\
\text { validation } \\
\text { error }(\%)\end{array}$ & $\begin{array}{c}\text { Accuracy } \\
(\%)\end{array}$ & $\begin{array}{c}\text { Error } \\
(\%)\end{array}$ & $\begin{array}{c}\text { Cross- } \\
\text { validation } \\
\text { error }(\%)\end{array}$ & $\begin{array}{c}\text { Accuracy } \\
(\%)\end{array}$ & $\begin{array}{l}\text { Error } \\
(\%)\end{array}$ & $\begin{array}{l}\text { Cross- } \\
\text { validation } \\
\text { error }(\%)\end{array}$ & $\begin{array}{c}\text { Accuracy } \\
(\%)\end{array}$ \\
\hline $\mathrm{C} 4.5$ & 0.68 & 7.31 & 92.69 & 2.37 & 8.41 & 91.59 & 3.05 & 8.62 & 91.38 \\
\hline Support vector machine & 8.81 & 10.34 & 89.66 & 14.92 & 15.10 & 84.90 & 20.00 & 20.34 & 79.66 \\
\hline Multilayer perceptron & 2.71 & 5.03 & 94.97 & 4.41 & 5.17 & 94.83 & 10.17 & 12.07 & 87.93 \\
\hline Bayes classifier & 7.80 & 8.55 & 91.45 & 12.54 & 13.03 & 86.97 & 16.95 & 18.00 & 82.00 \\
\hline Linear discriminant analysis & 4.75 & 6.14 & 93.86 & 9.83 & 11.79 & 88.21 & 14.58 & 16.07 & 83.93 \\
\hline
\end{tabular}

The lowest values of errors and the greatest accuracy are shown in bold.

expression of classifying miRNA to the NF4 (geometric mean of miR-197, -151a, -214 and -99a), reference miRNA characterized by the greatest expression stability (miR-197) and by the lowest expression stability (miR-99a).

In order to classify the samples by miRNA profiling we used methods belonging to different classes: Methods based on statistical procedures, i.e., Bayes classification algorithm and linear discriminant analysis and cybernetic methods: support vector machine and neural networks with backpropagation of error (multilayer perceptron); and the decision-tree algorithm (C4.5), belonging to the class of logical methods. The prediction quality assessment was based on the crossvalidation with 5 partitions. The classification was performed twice: in the first case the samples were subdivided into PTC, MTC and benign neoplasms, in the second case they were subdivided into malignant and benign. The comparison of the classification results was based on the percentage of errors, including the average error during cross-validation. The error was understood as a discrepancy between the cytology and miRNA classification diagnoses. The results are presented in Table III.

As expected, for all methods the lowest error was observed for data normalized to the NF4, in some cases, the observed decrease in the percentage of errors was 2-fold or greater. Making use of data normalized to miR-99a resulted in the greatest error. Data normalized to miR-197 yielded interme- diate value of error. There was only one deviation from this pattern, in case of classification PTC/MTC/Benign by decision-tree method the percentage of errors under normalization to miR-99a proved to be lower than under the normalization to miR-197 (Table III).

\section{Discussion}

The miRNA expression in tumors is always different from that in healthy tissue and at different stages of tumor development. Since miRNAs are highly stable in biological tissues and body fluids, this makes them promising diagnostic markers. However, the use of miRNAs as a reliable biomarker in oncology diagnostics is impossible without the proper normalization of quantitative data on their expression. The main purpose of the normalization is to reduce the technical variation of experimental data, which permits to perform a more accurate assessment of the biological variation. At present, there are a number of various studies devoted to the determination of profiles of miRNA expression for different thyroid pathologies. However, the numerous obtained data are not always confirmed in other studies and sometimes are even contradictory (31). This is usually attributed to the difference in platforms used for the quantification of miRNA expression, in different methods of miRNA isolation and peculiarities of the analyzed batches of samples. Nevertheless, use of 

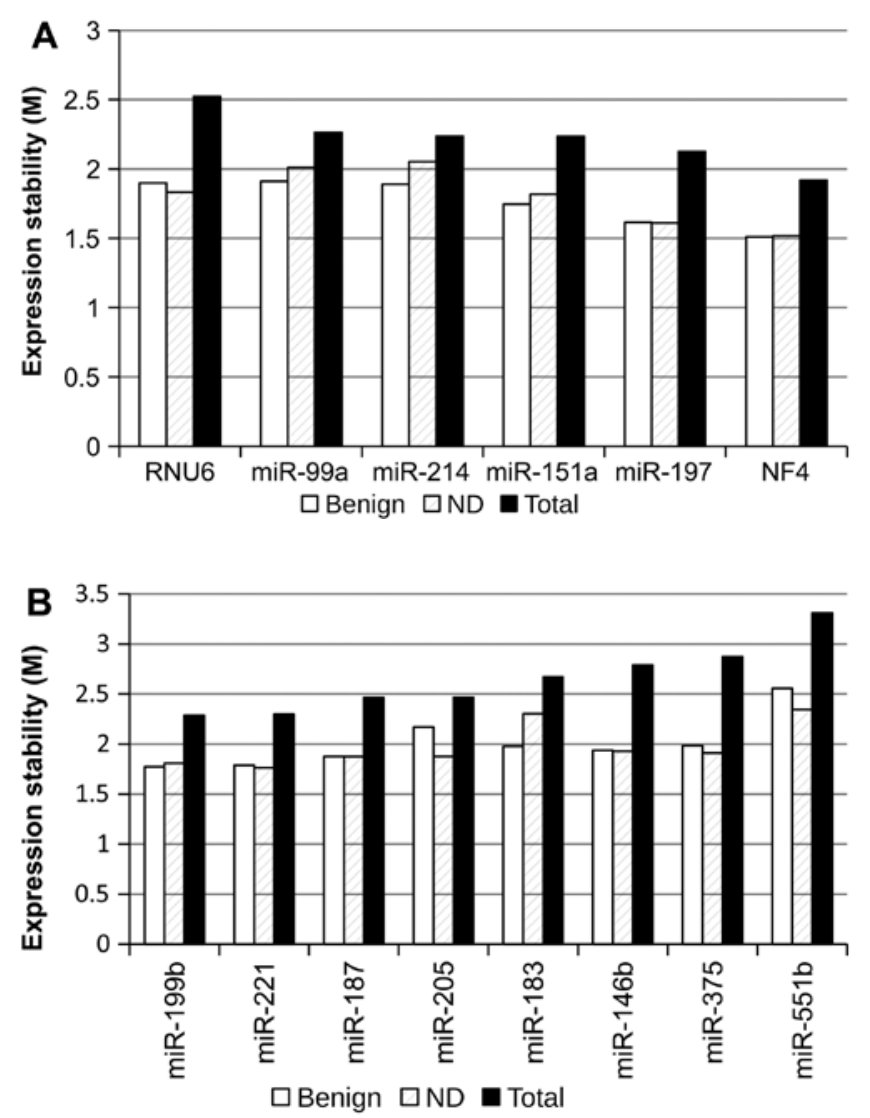

Figure 7. M-values for: (A) reference miRNAs, NF4 and RNU6; (B) miRNA classifiers. Black columns, the entire sampling of FNAC smears; shaded columns, non-diagnostic material; and blank columns, benign neoplasms.

improper reference genes can be one of the major reasons for the differences in the results obtained in studies of miRNA expression (14). Even slight changes in the content of miRNAs may be biologically significant but these changes may be estimated incorrectly or even remain hidden in the case of unsuccessful choice of normalization strategy $(14,32,33)$. Since there are no universal miRNA normalizers for various types of tissues and cells, every research requires the selection of suitable reference genes just for the specific tissue and type of material.

In the present study, we describe the choice of reference genes making it possible to compare the levels of miRNA expression in cytological thyroid smears dried on glass by RT-qPCR based methods. At the first stage the candidates were selected by chip technology from a small batch of samples; then the expression stability of the candidate genes was assessed on an larger batch of clinical samples using PCR; and at the third stage the selected variants were validated by including them in diagnostic algorithm and evaluating the contribution of the normalizer to the general error of the analysis. As a result, we chose four miRNA candidates and for comparison we used RNU6 as the commonly used normalizer. The evaluation of the expression stability showed that for the reference miRNAs it was higher than for the classifying ones and higher than for RNU6. As for the latter, its expression stability was at the same level as the most pronounced miRNA classifiers (miR-146b and -375). Moreover, the validation of normalizers revealed that the $\mathrm{C} 4.5$ algorithm, regardless on which refer- ence miRNA quantification data were normalized to, included RNU6 in the decision tree, i.e., assigns RNU6 to classifiers. The above suggests that RNU6 cannot be used as a normalizer in studying miRNA expression in thyroid tumors. Apparently, during the development of malignant tumors RNU6 expression changes in a characteristic way, so it can serve as a marker of the process, which has been shown for some other types of cancer $(15,34)$.

Using several reference genes instead of one has really made it possible to improve the quality of the data normalization. In order to compare the quality of normalization to different reference genes, we classified the obtained data using different algorithms and compared them with cytological classification. The algorithms employed by us, with the exception of the decision-tree method (C4.5) use the entire set of the data, therefore, data with the least general technical variability will give the best results. On constructing the decision tree, C4.5 algorithm selects data only from the miRNAs yielding the best classification and is, therefore, less dependent on the general variability. Our verification showed that making use of the complex normalizer consisting of four miRNAs for the data normalization results in the decrease percentage of both direct and cross-validation errors and this is observed regardless of the employed algorithm. With normalization to a single miRNA, making use of miRNA with the most stable expression give lower percentage of errors for all classification algorithms except C4.5. For the latter, this rule does not strictly apply, which suggests that in the selection of the normalizer we should take into account not only its stability, but also the methods of the further data analysis to be used.

It should be emphasized that in this study, we did not equalize the analyzed preparations with regard to nucleic acids concentration and did not control their degradation or purity. For the isolation of nucleic acids a standard low-cost method was used, which did not involve the purification of small RNA fraction. We used such an approach in order to simulate the real work carried out in low resource laboratories, without disproportionate labor costs associated with evaluation and equalization of nucleic acids concentration in each sample before miRNA quantification. Moreover, different slides in the studied sample were stored in archive for different time (1-5 years). The aim of the present study was not to obtain the minimum M-values, but rather to choose the set of normalizers suitable for the analysis of this particular clinical material, i.e. cytological preparations dried on glass slides. The comparison of results obtained from the cytological smears and the surgical material clearly indicates that the M-value depends on the variation in the content of the nucleic acids in the sample. As our experience shows, we should not expect too much from the material quality of dried FNAC samples. This type of material is characterized by a significant level of DNA degradation and, even more so, of RNA (especially when they are stored in archives), as well as great variability in the amount of sampled material and unwanted impurities therein (e.g., blood). Moreover, in our experience, these parameters can vary depending on the particular medical institution and skills of the laboratory staff in the setting where sampling is performed.

Another peculiarity of the sample we used to validate the normalizer was the fact that the sample was enriched with 
malignant neoplasms. We believe this approach enables more correct evaluation of the compared normalizers and makes it possible to choose the optimum variants on more rational grounds. In analyzing the sampling corresponding to the real flow of FNAC samples, it will likely consist mostly of benign smears. Then the stability of all analyzed short RNAs would be higher and the biological variation, characteristic of miRNA classifiers and enabling their use for thyroid neoplasms typing, would be less noticeable. When the M-values are only determined for preparations classified as benign neoplasms $(n=226)$ these values prove lower than for the entire sampling both for normalizers and for classifiers. The M-values close to benign samples have been obtained for the subgroup corresponding to non-diagnostic material and not used for the validation of normalizers $(n=34)$ (Fig. 7). The latter is probably due to the fact that the sampling of non-diagnostic material contains higher proportion of preparations from benign neoplasms, corresponding to their statistical occurrence in patients with suspected thyroid cancer.

It should be noted that the expression of all selected reference miRNAs may in fact be different for the compared groups of smears corresponding to different types of lesions. It is clear from the data presented in Table II that none of the selected reference markers has proved itself as universal in comparing different types of neoplasms. None of the suggested normalizers was able to distinguish non-diagnostic material from benign smears, which confirms our assumption that the sample of non-diagnostic smears primarily corresponds to benign neoplasms due to their high statistical representation. At the same time, miR-99a actually appears a 'weak' classifier distinguishing benign neoplasms from both types of cancer, miR-214 distinguishes the batch of PTCs from the other two groups, and miR-151a sorts out MTCs. It can be seen from the presented median $\mathrm{Cq}$ values that these differences cannot be attributed only to artifacts associated with varying degrees of nucleic acids degradation in the compared groups. Moreover, on average, samples of malignant neoplasms were longer stored in archives, especially samples of the rare medullary thyroid carcinomas, and it could be expected that the $\mathrm{Cq}$ values for those would be higher. Nevertheless, our data do not support this assumption.

According to the available data obtained by different methods, deregulation is demonstrated for all selected miRNA normalizers for some particular types of cancer. For example, upregulation is demonstrated for hsa-miR-197 in follicular thyroid carcinoma (35) and lung cancer (36), for hsa-miR-214 downregulation is shown for the cases of ovarian cancer (37) but it is upregulated in pancreatic carcinoma (38). All these miRNAs are controlled by key transcription factors and changes in their expression may be involved in the mechanism of malignant transformation.

We are not able to rule out the assumption that at least in the case of thyroid neoplasms none of miRNA genes is a housekeeping gene in the classical sense, that is, a gene, the expression of which is uniform in a heterogeneous sample comprising different types of neoplasms. Or, perhaps, such 'miRNA house-keepers' should be sought among lowexpressed miRNA genes which had been discarded by us because of their low expression and, consequently, high variation in measurements using PCR. At the same time, the use of the complex normalizer can compensate for distortion in quantitative evaluation introduced by individual miRNAs in its composition, which are expressed differently in different groups of samples. In our case, the fact that the complex normalizer gives a lower error rate than any of the individual reference genes in its composition, indirectly confirms this assumption.

Thus, we have proposed the normalization factor (geometric mean values of the content of miR-151a-3p, -197$3 p,-99 a-5 p$ and $-214-3 p$ ), which can be used in the analysis of changes in the content of miRNA in dried cytological smears from thyroid lesions. In our hands, the use of this normalizer, despite the substantial variation in the content of the nucleic acids and the biological variation of the levels of reference miRNAs, made it possible to discriminate different types of thyroid lesions in cytological preparations with a fairly high total accuracy, using a simple classifier including a limited number of miRNAs.

\section{Acknowledgements}

The present study was supported by the Fundamental Scientific Research on the project 0310-2015-0003,0310-2015-0007 (NNK and SET) and 0324-2015-0003 (PSD).

\section{References}

1. Guth S, Theune U, Aberle J, Galach A and Bamberger CM: Very high prevalence of thyroid nodules detected by high frequency $(13 \mathrm{MHz})$ ultrasound examination. Eur J Clin Invest 39: 699-706, 2009.

2. Orlandi A, Puscar A, Capriata E and Fideleff H: Repeated fineneedle aspiration of the thyroid in benign nodular thyroid disease: Critical evaluation of long-term follow-up. Thyroid 15: 274-278, 2005.

3. Stevens C, Lee JK, Sadatsafavi M and Blair GK: Pediatric thyroid fine-needle aspiration cytology: A meta-analysis. J Pediatr Surg 44: 2184-2191, 2009.

4. Witt RL, Ferris RL, Pribitkin EA, Sherman SI, Steward DL and Nikiforov YE: Diagnosis and management of differentiated thyroid cancer using molecular biology. Laryngoscope 123: 10591064, 2013

5. Pallante P, Visone R, Ferracin M, Ferraro A, Berlingieri MT, Troncone G, Chiappetta G, Liu CG, Santoro M, Negrini M, et al: MicroRNA deregulation in human thyroid papillary carcinomas. Endocr Relat Cancer 13: 497-508, 2006.

6. Kitano M, Rahbari R, Patterson EE, Steinberg SM, Prasad NB, Wang Y, Zeiger MA and Kebebew E: Evaluation of candidate diagnostic microRNAs in thyroid fine-needle aspiration biopsy samples. Thyroid 22: 285-291, 2012.

7. Swierniak M, Wojcicka A, Czetwertynska M, Stachlewska E, Maciag M, Wiechno W, Gornicka B, Bogdanska M, Koperski L, de la Chapelle A, et al: In-depth characterization of the microRNA transcriptome in normal thyroid and papillary thyroid carcinoma. J Clin Endocrinol Metab 98: E1401-E1409, 2013.

8. Cancer Genome Atlas Research Network: Integrated genomic characterization of papillary thyroid carcinoma. Cell 159: 676-690, 2014.

9. Meyer SU, Pfaffl MW and Ulbrich SE: Normalization strategies for microRNA profiling experiments: A 'normal' way to a hidden layer of complexity? Biotechnol Lett 32: 1777-1788, 2010.

10. Huggett J, Dheda K, Bustin S and Zumla A: Real-time RT-PCR normalisation; strategies and considerations. Genes Immun 6: 279-284, 2005

11. Bustin SA, Benes V, Garson JA, Hellemans J, Huggett J, Kubista M, Mueller R, Nolan T, Pfaffl MW, Shipley GL, et al: The MIQE guidelines: Minimum information for publication of quantitative real-time PCR experiments. Clin Chem 55: 611-622, 2009.

12. Spanakis E: Problems related to the interpretation of autoradiographic data on gene expression using common constitutive transcripts as controls. Nucleic Acids Res 21: 3809-3819, 1993. 
13. Vandesompele J, De Preter K, Pattyn F, Poppe B, Van Roy N, De Paepe A and Speleman F: Accurate normalization of real-time quantitative RT-PCR data by geometric averaging of multiple internal control genes. Genome Biol 3: H0034, 2002.

14. Dijkstra JR, van Kempen LC, Nagtegaal ID and Bustin SA: Critical appraisal of quantitative PCR results in colorectal cancer research: Can we rely on published qPCR results? Mol Oncol 8: 813-818, 2014.

15. Gee HE, Buffa FM, Camps C, Ramachandran A, Leek R, Taylor M, Patil M, Sheldon H, Betts G, Homer J, et al: The smallnucleolar RNAs commonly used for microRNA normalisation correlate with tumour pathology and prognosis. Br J Cancer 104: 1168-1177, 2011

16. Appaiah HN, Goswami CP, Mina LA, Badve S, Sledge GW Jr, Liu Y and Nakshatri H: Persistent upregulation of U6:SNORD44 small RNA ratio in the serum of breast cancer patients. Breast Cancer Res 13: R86, 2011.

17. Cibas ES and Ali SZ; NCI Thyroid FNA State of the Science Conference: The Bethesda System For Reporting Thyroid Cytopathology. Am J Clin Pathol 132: 658-665, 2009.

18. Titov SE, Ivanov MK, Karpinskaya EV, Tsivlikova EV, Shevchenko SP, Veryaskina YA, Akhmerova LG, Poloz TL, Klimova OA, Gulyaeva LF, et al: miRNA profiling, detection of BRAF V600E mutation and RET-PTC1 translocation in patients from Novosibirsk oblast (Russia) with different types of thyroid tumors. BMC Cancer 16: 201, 2016.

19. Ricco R: TANAGRA: a free software for research and academic purposes. In: Proceedings of EGC'2005, RNTI-E-3, vol. 2, pp.697-702, 2005 (in French).

20. Rao RC: The utilization of multiple measurements in problems of biological classification. J R Stat Soc B 10: 159-203, 1948.

21. Hand DJ and Yu Y: Idiots Bayes - not so stupid after all? Int Stat Rev 69: 385-389, 2001.

22. Rumelhart DE, McClelland JL and the PDP Research Group: Parallel distributed processing: Explorations in the microstructure of cognition I. MIT Press, Cambridge, MA, 1986.

23. Chang CC and Lin CJ: LIBSVM: a library for support vector machines. ACM Trans Intell Syst Technol 2 (3, article 27): 1-39, 2011. 10.1145/1961189.1961199.

24. Quinlan JR: C4.5: programs for machine learning. Morgan Kaufmann Publishers Inc., San Francisco, CA, 1993.

25. Kulkarni MM: Digital multiplexed gene expression analysis using the NanoString nCounter system. Curr Protoc Mol Biol 94: 25B.10.1-25B.10.17, 2011

26. Nikiforova MN, Tseng GC, Steward D, Diorio D and Nikiforov YE: MicroRNA expression profiling of thyroid tumors: Biological significance and diagnostic utility. J Clin Endocrinol Metab 93: 1600-1608, 2008.
27. Abraham D, Jackson N, Gundara JS, Zhao J, Gill AJ, Delbridge L, Robinson BG and Sidhu SB: MicroRNA profiling of sporadic and hereditary medullary thyroid cancer identifies predictors of nodal metastasis, prognosis, and potential therapeutic targets. Clin Cancer Res 17: 4772-4781, 2011.

28. Rossing M, Borup R, Henao R, Winther O, Vikesaa J, Niazi O, Godballe C, Krogdahl A, Glud M, Hjort-Sørensen C, et al: Downregulation of microRNAs controlling tumourigenic factors in follicular thyroid carcinoma. J Mol Endocrinol 48: 11-23, 2012.

29. Dettmer M, Perren A, Moch H, Komminoth P, Nikiforov YE and Nikiforova MN: Comprehensive MicroRNA expression profiling identifies novel markers in follicular variant of papillary thyroid carcinoma. Thyroid 23: 1383-1389, 2013.

30. Hellemans J and Vandesompele J: Selection of reliable reference genes for RT-qPCR analysis. In: Quantitative Real-Time PCR: Methods and Protocols, Methods in Molecular Biology. Vol. 1160. Biassoni R and Raso A (eds). Springer Science Business Media, New York, pp19-26, 2014.

31. Pallante P, Battista S, Pierantoni GM and Fusco A: Deregulation of microRNA expression in thyroid neoplasias. Nat Rev Endocrinol 10: 88-101, 2014.

32. Bargaje R, Hariharan M, Scaria V and Pillai B: Consensus miRNA expression profiles derived from interplatform normalization of microarray data. RNA 16: 16-25, 2010.

33. Shen Y, Li Y, Ye F, Wang F, Wan X, Lu W and Xie X: Identification of miR-23a as a novel microRNA normalizer for relative quantification in human uterine cervical tissues. Exp Mol Med 43: 358-366, 2011.

34. Thorenoor $\mathrm{N}$ and Slaby O: Small nucleolar RNAs functioning and potential roles in cancer. Tumour Biol 36: 41-53, 2015.

35. Weber F, Teresi RE, Broelsch CE, Frilling A and Eng C: A limited set of human MicroRNA is deregulated in follicular thyroid carcinoma. J Clin Endocrinol Metab 91: 3584-3591, 2006.

36. Du L, Schageman JJ, Subauste MC, Saber B, Hammond SM, Prudkin L, Wistuba II, Ji L, Roth JA, Minna JD, et al: miR-93, miR-98, and miR-197 regulate expression of tumor suppressor gene FUS1. Mol Cancer Res 7: 1234-1243, 2009.

37. Yang H, Kong W, He L, Zhao JJ, O'Donnell JD, Wang J, Wenham RM, Coppola D, Kruk PA, Nicosia SV, et al: MicroRNA expression profiling in human ovarian cancer: miR-214 induces cell survival and cisplatin resistance by targeting PTEN. Cancer Res 68: 425-433, 2008.

38. Zhang XJ, Ye H, Zeng CW, He B, Zhang $\mathrm{H}$ and Chen $\mathrm{YQ}$ : Dysregulation of miR-15a and miR-214 in human pancreatic cancer. J Hematol Oncol 3: 46, 2010. 